\title{
Errores de administración de medicación en los servicios de urgencias
}

\section{Medication administration errors in emergency departments}

\author{
S. Tomás Vecina ${ }^{1,4}$, M. Chanovas Borrás ${ }^{2,4}$, F. Roqueta Egea ${ }^{3,4}$
}

\section{Sr. Director:}

Recientemente, Ambrosio y col han publicado un trabajo en su revista en donde se analiza los factores del entorno de trabajo que influyen en la ocurrencia de errores en la administración de medicación (EAM), basado en la revisión de la literatura a partir de la consulta de bases de datos bibliográficas internacionales entre 2002 y $2012^{1}$.

Debido a que la metodología seguida por los autores no permite incluir en su análisis los estudios que han sido realizados en otros ámbitos asistenciales, creemos de interés aportar nuestra experiencia, desde el Programa SEMES-Seguridad Paciente $^{2}$ sobre los EAM en el entorno de la atención urgente hospitalaria, como una información complementaria que puede reforzar y/o aportar más datos al respecto y de la seguridad del paciente (SP).

En este sentido, y dentro del mismo periodo de análisis, el programa SEMES-SP ha desarrollado tres experiencias que analizan directa o indirectamente los EAM:

1. El estudio EVADUR (Eventos Adversos en Urgencias) $)^{3}$, en donde se identificó que, al menos, un $12 \%$ de los pacientes atendidos en Urgencias tuvo un incidente de SP, sin o con daño (evento adverso). En dicho estudio, el $37,6 \%$ de los incidentes detectados tuvo como factor causal algún tipo de problema relacionado con el manejo de la medicación (PRM), de los que el 13\% no llegaron a causar daño y cerca del $25 \%$ restante fue un evento adverso (EA). En ellos los EAM estuvieron implicados en casi el 50\% de dichos EA.

2. El desarrollo de un Mapa de Riesgos de la Atención Urgente ${ }^{4}$, validado entre 23 hospitales españoles, en donde se identifican 1.368 situaciones posibles de riesgos durante el proceso asistencial urgente, de los que 192 (14\%) corresponden al proceso de tratamiento, y de éstos, 98 (51\%) al subproceso de administración Entre ellos destaca que, al analizar la mediana de la puntuación del índice de probabilidad de riesgos (IPR) entre los hospitales participantes, 5 de los 12 mayores riesgos estaban relacionados con la administración de medicación (Tabla 1). El factor de entorno laboral implicado más frecuente en la validación realizada entre todos los participantes fue la sobrecarga asistencial (10\%).

3. El apoyo a la figura del farmacéutico en los servicios de Urgencias como una estrategia esencial para reducir los problemas de seguridad derivados del empleo de medicación en urgencias ${ }^{2,5}$, estrategia reforzada junto con la publicación de un documento de consenso sobre Conciliación de Mediación realizado entre la RedFaster de la Sociedad Española de Farmacia Hospitalaria y SEMES ${ }^{6}$.

En los SU, los principales errores de medicación se generan en las fases de prescripción y administración. La situación clínica del paciente, el elevado número de fármacos empleados en poco espacio de tiempo, las frecuentes interferencias e

An. Sist. Sanit. Navar. 2013; 36 (3): 539-541

1. Hospital Municipal de Badalona.

2. Hospital Virgen de la Cinta. Tortosa.

3. Fundación Althaia. Manresa.

4. Programa SEMES-Seguridad Paciente.

\section{Correspondencia}

Santiago Tomás Vecina

C/ Rosselló, $3276^{\circ}$

08037 Barcelona

santitomas@ono.com 
Tabla 1. Proceso de tratamiento en los servicios de Urgencias: riesgos más importantes*

\begin{tabular}{|c|c|}
\hline Riesgos y causa & IPR* * \\
\hline Selección de un fármaco inadecuado por anamnesis incorrecta & 372 \\
\hline Selección de un fármaco inadecuado por diagnóstico incorrecto & 400 \\
\hline Selección de un fármaco inadecuado por sobrecarga asistencial & 445 \\
\hline Elección incorrecta de un fármaco por prescripción verbal incorrecta & 400 \\
\hline Demora en la administración de un fármaco por sobrecarga asistencial & 372 \\
\hline No verificación tratamiento previa administración (paciente, dosis, vía, fármaco) por sobrecarga asistencial & 372 \\
\hline Administración incorrecta de un fármaco por error de dosis & 400 \\
\hline Administración incorrecta de un fármaco por concentración inadecuada & 372 \\
\hline Retraso en la administración de un tratamiento por sobrecarga asistencial en el área observación & 400 \\
\hline Deficiente monitorización de la eficacia del tratamiento por distracción/olvido/interrupciones & 400 \\
\hline Ausencia de monitorización de la eficacia del tratamiento por sobrecarga asistencial & 372 \\
\hline Información inadecuada sobre el tratamiento y seguimiento domiciliario al alta & 400 \\
\hline
\end{tabular}

interrupciones, y las condiciones laborales, especialmente de enfermería, colectivo responsable de la administración, se involucran como factores responsables ${ }^{7,8}$. Coincidimos con los autores en las conclusiones y propuestas sugeridas para reducir los EAM en el entorno sanitario, pero, en el campo concreto de los SU nos permitimos resaltar, en base a nuestra experiencia, que es prioritaria, al menos, la introducción de estrategias dirigidas a reducir los EAM de fármacos de alto riesgo por el elevado peligro de EA que pueden generar ${ }^{9}$, así como la importación de experiencias organizativas con éxito en otros ámbitos para reducir las interrupciones ("non disturb please") ${ }^{10}$.

\section{BIBLIOGRAFÍA}

1. Ambrosio L, Pumar-MÉndez MJ. Factores del entorno de trabajo que influyen en la ocurrenciade errores de administración de medicación. An Sist Sanit Navar 2013; 36: 77-85.

2. Tomás S, Chanovas M, Rogueta C, Toranzo T. Patient safety in emergency medicine: report on 4 years' implementation of a program designed for the Spanish Society of Emergency Medicine (SEMES). Emergencias 2012; 24: 225-233.

3. Tomás S, Chanovas M, Roqueta F, Alcaraz J, Toranzo T y Grupo de Trabajo EVADUR-SEMES. EVADUR: eventos adversos ligados a la asistencia en los servicios de urgencias de hospitales españoles. Emergencias 2010; 22: 415-428.

4. Tomás Vecina S, Bueno Domínguez MJ, Chanovas BoRRÁs M, RoQueta Egea F y Grupo de Trabajo Mapa de Riesgos SEMES. Diseño y validación de un mapa de riesgos para la mejora de la Seguridad del Paciente en los Servicios de Urgencias Hospitalarios. Trauma 2013 (en prensa).

5. Tomás Vecina S, García Sanchez L, Pacual Arce B, RiERA PAREDEs I. Programa de intervención farmacéutica en el Servicio de Urgencias para mejorar la seguridad del paciente. Emergencias 2010; 22: 85-90.

6. Calderón Hernánz B, Oliver Noguera A, Tomás Vecina S, Baena Parejo Mi, García Peláez M, Juanes Borrego A et al. Conciliación de los medicamentos en los Servicios de Urgencias. Emergencias 2013; 25: 204-217.

7. Patanwala AE, Warholak TL, Sanders AB, Erstad BL. A prospective observational study of medication errors in a tertiary care emergency department. Ann Emerg Med 2010; 55: 522-526.

8. Tomás S, Gimena I. La seguridad del paciente en urgencias y emergencias. An Sist Sanit Navar 2010; 33 (Supl. 1): 131-148.

9. Lista de Medicamentos de Alto Riesgo. ISMP-España. Septiembre 2012. (consultado 12-08-2013). Disponible en: http://www.ismp-espana.org/estaticos/view/39

10. Anthony K, Wiencek C, Bauer C, Daly B, Anthony MK. No interruptions please: impact of a no interruption zone on medication safety in intensive care units. Crit Care Nurse 2010; 30: 21-29. 


\title{
Errores de administración de medicación en los servicios de urgencias
}

\author{
L. Ambrosio, M.J. Pumar-Méndez
}

Nos gustaría agradecer los comentarios ofrecidos sobre nuestro trabajo "Factores del entorno de trabajo que influyen en la ocurrencia de errores de administración de medicación", a los autores de la carta al editor enviada a esta revista recientemente. Consideramos que el debate que genera es muy enriquecedor para el desarrollo de esta área tan relevante.

Las interesantes experiencias citadas por nuestros colegas se centran en el campo de la atención urgente hospitalaria. Aunque nuestra revisión no incluyó dicho ámbito, creemos que una ampliación del trabajo a este campo sería muy interesante. No en vano, el ámbito de la atención urgente hospitalaria reúne las características definitorias de los "sistemas complejos", y en consecuencia, constituyen servicios altamente proclives a la ocurrencia de errores clínicos como son los errores de administración de medicación ${ }^{1}$.

Coincidimos enteramente con los autores del comentario de nuestro trabajo en que la idiosincrasia del entorno de la atención urgente hospitalaria debe ser tenida en cuenta a la hora de proponer acciones dirigidas a la reducción de errores de administración de medicación. Así mismo, estamos de acuerdo en que los esfuerzos dirigidos a reducir los EAM de fármacos de alto riesgo deben ser priorizados, principalmente dado el gran impacto que estos últimos pueden tener en los pacientes. En este sentido, la adaptación al ámbito de la atención urgente hospitalaria de listados de verificación para la administración de fármacos de alto riesgo puede constituir una medida específica y costo-efectiva ${ }^{2}$. Sin embargo, nos parece importante resaltar que otras estrategias más genéricas (o enfocadas a mejorar factores del entorno de trabajo que influyen en la ocurrencia de EAMs de todo tipo y no únicamente de fármacos de alto riesgo), pueden ser igualmente efectivas en la reducción de los EAM de fármacos de alto riesgo, con el valor añadido de que otros tipos de EAMs pueden ser prevenidos de modo adyacente.

La prioridad identificada por nuestros colegas de "importar experiencias organizativas con éxito" nos parece especialmente oportuna. La adaptación contextual de medidas que ya cuentan con evidencia de su efectividad (aunque ésta sea preliminar en algunos casos) debe prevalecer. De este modo se generará, de un modo mucho más rápido y comparable, conocimiento sobre estrategias para la gestión de factores del entorno que contribuyen a los EAMs ${ }^{3}$. En este sentido, sugerimos que se comience por adaptar aquellas estrategias para la gestión de factores del entorno contribuyentes a los EAMs que parecen ser comunes para ámbitos diversos como el de la atención urgente hospitalaria y las unidades de hospitalización. Éstos, como bien resaltan nuestros colegas, son: las distracciones, las interrupciones, y la sobrecarga de trabajo.

\section{BIBLIOGRAFÍA}

1. Perrow C. Normal Accidents: Living with HighRisk Technology. Princeton University Press: Princeton. 1984

2. White RE, Trbovich Pl, Easty AC, Savage P, Trip K, Hyland S. Checking it twice: an evaluation of checklists for detecting medication errors at the bedside using a chemotherapy model. Qual Saf Health Care 2010; 19: 562-567.

3. Houser J, Oman K S. Evidence-Based Practice. An Implementation Guide for Healthcare Organizations. Burlington: Jones \& Bartlett Publishers. 2011.

\section{Correspondencia}

Leire Ambrosio Gutiérrez

Dpto. de Enfermería de la Persona Adulta

Facultad de Enfermería

Universidad de Navarra

31008 Pamplona

lambrosio@unav.es 